doi:10.13108/2015-7-1-58

UDC 517.925

\title{
STABILITY OF AUTORESONANCE IN DISSIPATIVE SYSTEMS
}

\author{
O.A. SULTANOV
}

\begin{abstract}
We consider a mathematical model describing the initial stage of a capture into autoresonance in nonlinear oscillating systems with a dissipation. Solutions whose amplitude increases unboundedly in time correspond to a resonance. An asymptotic expansion for such solutions is constructed as a power series with constant coefficients. The stability of autoresonance with respect to persistent perturbations is studied by means of Lapunov's second method. We describe the classes of perturbations for which a capture into autoresonance occurs.
\end{abstract}

Keywords: resonance, nonlinear oscillations, dissipation, perturbations, stability.

Mathematics Subject Classification: 34D05, 70K30, 93D05, 93D20

\section{INTRODUCTION}

Formulation of problem. We consider a mathematical model describing the initial stage of capture into autoresonance [1, 2] in nonlinear oscillating systems with a small pumping [3] and dissipation:

$$
\frac{d r}{d \tau}=f(\tau) \sin \psi-\beta r, \quad r\left[\frac{d \psi}{d \tau}-r^{2}+\lambda \tau\right]=g(\tau) \cos \psi, \quad \tau>0, \quad \lambda, \beta=\text { const }>0 .
$$

The sought functions $r(\tau)$ and $\psi(\tau)$ correspond to a slowly changing amplitude and a phase shift of a fast harmonic oscillation. Autoresonance is usually associated to solutions with an unboundedly growing amplitude $r(\tau) \approx \sqrt{\lambda \tau}$. Variable coefficients $f(\tau)=f_{0}+f_{1} \tau, g(\tau)=$ $g_{0}+g_{1} \tau\left(f_{1}, g_{1} \neq 0\right)$ are responsible for the amplitude of pumping. Due to the invariance of the system w.r.t. the change $\psi \Rightarrow \psi+\pi, f, g \Rightarrow-f,-g$, without loss of generality we can assume $f_{1}<0$. Parameter $\beta$ corresponds to the dissipation coefficient.

System (1) emerges as the result of averaging in the small parameter [4] of nonlinear nonisochronous oscillations with a pumping of small amplitude. As an example we consider Duffing equation with dissipation

$$
\begin{gathered}
\frac{d^{2} x}{d s^{2}}+x-\gamma x^{3}=-\delta \frac{d x}{d s}+\varepsilon A(s ; \varepsilon) \cos \phi(s ; \alpha) \\
A(s ; \varepsilon)=A_{0}+A_{1}(\varepsilon) s, \quad \phi(s ; \alpha)=s-\alpha s^{2}, \quad 0<\alpha, \delta, \varepsilon \ll 1, \quad \gamma>0 .
\end{gathered}
$$

Asymptotic ansätze

$$
x(s ; \varepsilon)=\varepsilon^{1 / 3} \kappa \cdot r(\tau) \cos [\phi(s ; \alpha)+\psi(\tau)]+\mathcal{O}\left(\varepsilon^{2 / 3}\right), \quad \varepsilon \rightarrow 0, \quad \tau=\varepsilon^{2 / 3} s,
$$

for solutions to equation (2) leads us to system (11) with the coefficients $\lambda=2 \alpha \varepsilon^{-4 / 3}, \beta=$ $\delta \varepsilon^{-2 / 3} / 2, f_{1}=g_{1}=-\varepsilon^{-2 / 3} A_{1} / 2 \kappa, f_{0}=g_{0}=-A_{0} / 2 \kappa, \kappa=2 \sqrt{2 / 3 \gamma}$. We note that in the

O.A. Sultanov, Stability of autoresonance in Dissipative systems.

(c) Sultanov O.A. 2015.

The research was supported by Russian Science Foundation (project no. 14-11-00078).

Submitted June 23, 2014. 
present problem, while studying the stability of solutions to system (11) the smallness of the coefficients of the equation is not employed.

Let us clarify the features of the considered problem, in particular, the choice of a special pumping in (2). We observe that the trajectories of the unperturbed equation $\ddot{x}+x-\gamma x^{3}=0$ with initial energy $0<E<1 /(4 \gamma)$ describe non-isochronous oscillations. An essential growth of the energy of such oscillations generated by a small $(0<\varepsilon \ll 1)$ oscillating force under the condition $\left|x\left(s_{0}\right)\right|+\left|\dot{x}\left(s_{0}\right)\right| \ll 1$ is usually associated with the autoresonance. Since our system is non-isochronous, a pumping with a constant frequency $(\alpha=0)$ does not produce an essential growth of oscillations amplitude [5]. It is obvious that one needs some adjustment of external force to a changing eigenfrequency. Such adjustment happens to be provided by a small change of pumping frequency. In particular, if $0<\alpha \ll 1, \delta=0$ and $A_{1}(\varepsilon)=0$, solutions to equation (2) can demonstrate an essential growth of energy (Fig. 1). In this case one says that a capture of an oscillating system into autoresonance occurs. We observe that the phenomenon of autoresonance or self-phasing has many applications [6, 7, 8, 9].

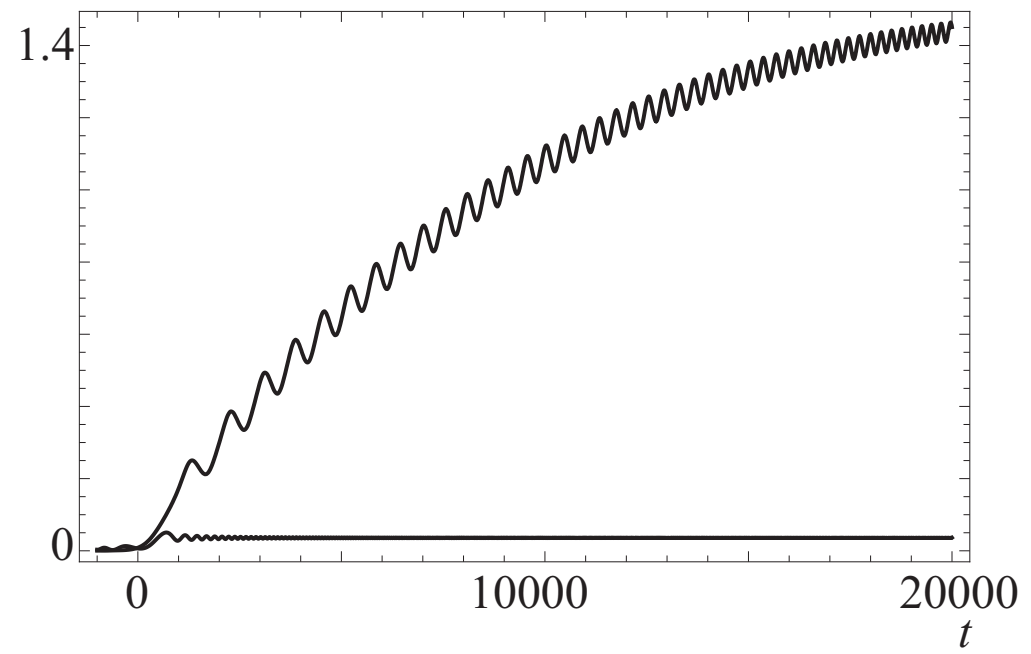

Fig. 1. Evolution of the energy of equation (2) with a variable frequency of pumping without dissipation: $\gamma=1 / 6, \delta=0, \varepsilon=10^{-3}, A_{0}=1, A_{1}=0, \alpha=10^{-5}$. Two graphs correspond to the solutions of different kinds captured into resonance and non-resonance.

Under the presence of the dissipation $(0<\delta \ll 1)$, a slowly changing force with $A(s ; \varepsilon) \equiv A_{0}$ produce a growth of oscillations only on a relatively small time interval, afterward the oscillations decay [10], Fig. 2a. A relatively long growth of the oscillation energy can be obtained by means of a small change of the pumping amplitude $A(s ; \varepsilon)=-2 \kappa\left(1+\varepsilon^{2 / 3} s\right)$, see Fig. $2 \mathrm{~b}$.

Such way of pumping for capturing into autoresonance of nonlinear oscillating system with dissipation was proposed in [3], where an asymptotic analysis of system (1) was made and there were obtained conditions of existence of a two-parametric family of solutions with a growing amplitude.

Autoresonance solution. For considered equations (1) we can obtain no explicit solutions. However, we can construct a formal asymptotic solution at infinity w.r.t. $\tau$ as a power series with constant coefficients:

$$
R_{0}(\tau)=\sqrt{\lambda \tau}+\sum_{k=0}^{\infty} r_{k} \tau^{-k / 2}, \quad \Psi_{0}(\tau)=\sum_{k=0}^{\infty} \psi_{k} \tau^{-k / 2}, \quad \tau \rightarrow \infty .
$$

Coefficients $r_{k}, \psi_{k}$ are determined by recurrent formulae after substituting the series in the equations. In this way we determine two solutions distinguished by the choice of a root to

\footnotetext{
${ }^{1}$ i.e., oscillations with the eigenfrequency depending on the amplitude.
} 
the equation $\sin \psi_{0}=0$. The existence of partial solutions as $\tau \geqslant \tau_{0}, \tau_{0}=$ const $>0$ with constructed asymptotics is implied by [11]. It follows from [12] that these solutions can be continued on the whole half-line $\tau \geqslant 0$. Below we discuss the stability of such solutions.

(a)

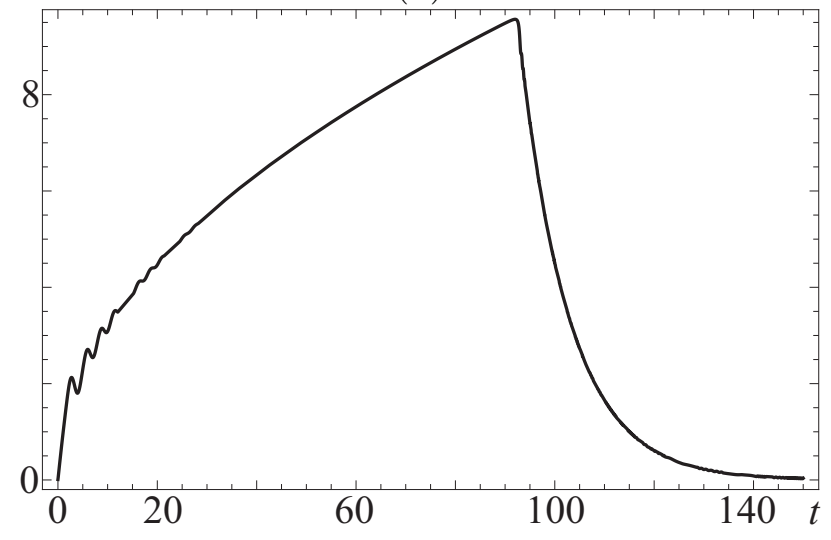

(b)

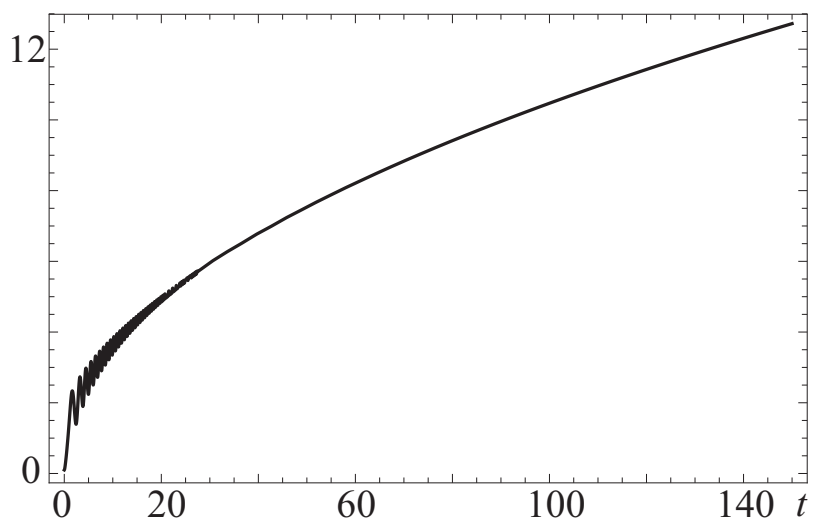

Fig. 2. Evolution of amplitude $r(\tau)$ as $\lambda=1, \beta=0.1$, (a): $f(\tau) \equiv g(\tau) \equiv 1$; (b): $f(\tau) \equiv g(\tau) \equiv-(1+\tau)$

Perturbed equations of principle resonance. The aim of the work is to study the influence of persistent perturbations on the capture into autoresonance. In order to do it, we consider perturbed equations:

$$
\frac{d r}{d \tau}=(f(\tau)+\mu \xi) \sin \psi-\beta r, \quad r\left[\frac{d \psi}{d \tau}-r^{2}+\lambda \tau+\mu \varphi\right]=(g(\tau)+\mu \eta) \cos \psi
$$

Here functions $\xi(r, \psi, \tau), \eta(r, \psi, \tau), \varphi(r, \psi, \tau)$ play the role of perturbations, $\mu \in \mathbb{R},|\mu| \ll 1$ is a small parameter controlling the perturbation [13].

An example of original problem averaging of which leads one to system (4) is the Duffing equation with perturbed pumping amplitude and phase:

$$
\frac{d^{2} x}{d s^{2}}+x-\gamma x^{3}=-\delta \frac{d x}{d s}+\varepsilon(A+\mu h) \cos (\phi+\mu \theta) .
$$

The perturbation is modeled by functions $h(x, \dot{x}, s ; \varepsilon)$ and $\theta(x, \dot{x}, s ; \varepsilon)$. In the particular case, when the perturbation depends on time only, the relation between functions $\xi(\tau), \eta(\tau), \varphi(\tau)$ and $h(s ; \varepsilon), \theta(s ; \varepsilon)$ is described by the following formulae:

$$
\xi(\tau)=a_{1} h(s ; \varepsilon), \quad \eta(\tau)=a_{2} h(s ; \varepsilon), \quad \varphi(\tau)=\theta^{\prime}(s ; \varepsilon) \varepsilon^{-2 / 3},
$$

where $a_{1}, a_{2}$ are some constants.

For model system (1) the problem of autoresonance stability is to identify a class of perturbations under which growing solution (3) is stable. In the present work we restrict ourselves by classes of perturbations under which system (4) has a global solution with initial data in the vicinity of solutions (3). Sufficient conditions ensuring this property are well-known (cf. [14], [15]).

We note that the models of autoresonance without dissipation were studied a lot including the stability (see [16, 17, 18]). However, in real physical processes there are always dissipation phenomena [19], which should be taken into consideration together with perturbations in the mathematical models. In the present work we propose a solution to problem of stability of autoresonance in nonlinear oscillating systems with a dissipation.

The work consists of three parts. In the first part we discuss the Lyapunov stability of autoresonance solutions w.r.t. initial data and we construct Lyapunov function. In the second part of the work we study the stability of autoresonance under persistent perturbations. The 
third part is devoted to the stability of autoresonance on an asymptotically long time interval. The main results of the work are the proof of autoresonance stability and the description of the classes of pertubations under which the capture into autoresonance occurs.

\section{LYAPUNOV STABILITY}

System (11) has a pair of solutions with asymptotics (3) distinguished by the choice of a root to the equation: $\sin \psi_{0}=0$. The solution with $\psi_{0}=\pi$ happens to be unstable that follows from the analysis of the eigenvalues of the matrix linearized on solution $R_{0}(\tau), \Psi_{0}(\tau)$ to the system.

For the other solution determined by the choice $\psi_{0}=0$, the eigenvalues of the linearized system are pure imaginary. Therefore, to study the stability we need to take into consideration nonlinear terms. We study the stability of this solution by Lyapunov's second method.

We precise asymptotics (3) of the considered solution as $\tau \rightarrow \infty$ :

$$
\begin{gathered}
R_{0}(\tau)=\sqrt{\lambda \tau}+r_{0}+r_{1} \tau^{-1 / 2}+\mathcal{O}\left(\tau^{-1}\right), \quad \Psi_{0}(\tau)=\psi_{1} \tau^{-1 / 2}+\mathcal{O}\left(\tau^{-1}\right), \\
r_{0}=-\frac{g_{1}}{2 \lambda}, \quad r_{1}=-\frac{g_{1}^{2}}{8 \lambda^{5 / 2}}, \quad \psi_{1}=\frac{\beta \lambda^{1 / 2}}{f_{1}} .
\end{gathered}
$$

We have

Theorem 1. Suppose that the coefficients of system (1) satisfy inequality $1+g_{1} / f_{1}>0$. Then solution $R_{0}(\tau), \Psi_{0}(\tau)$ with asymptotics (7) is asymptotically stable.

Proof. In system (1) we make a change

$$
r=R_{0}(\tau)+\nu R \tau^{1 / 4}, \quad \psi=\Psi_{0}(\tau)+\Psi, \quad t=\left(\frac{\tau}{z}\right)^{7 / 4}
$$

$\nu=\sqrt{-f_{1}} /(4 \lambda)^{1 / 4}, z=\left(-7 \nu / 4 f_{1}\right)^{4 / 7}$, and for new functions $R(t), \Psi(t)$ we study the stability of the equilibrium $(0 ; 0)$. In new variables original equations (1) cast into the form

$$
\frac{d R}{d t}=-\partial_{\Psi} H(R, \Psi, t), \quad \frac{d \Psi}{d t}=\partial_{R} H(R, \Psi, t)+G(R, \Psi, t) .
$$

Here Hamiltonian and non-Hamiltonian component read as: 1 :

$$
\begin{aligned}
H= & \frac{R_{0}(\tau)}{2 \sqrt{\lambda \tau}} R^{2}+\left(1+\frac{f_{0}}{f_{1}} \tau^{-1}\right)\left(\cos \Psi_{0}-\cos \left(\Psi+\Psi_{0}\right)-\Psi \sin \Psi_{0}\right)+ \\
& +\frac{\nu R^{3}}{6 \sqrt{\lambda}} \tau^{-1 / 4}+\frac{\left(4 \beta+\tau^{-1}\right) R \Psi}{8 \nu \sqrt{\lambda}} \tau^{-3 / 4} \\
G= & \frac{1}{2 \nu \sqrt{\lambda}}\left[g(\tau)\left(\frac{\cos \left(\Psi+\Psi_{0}\right)}{R_{0}(\tau)+\nu R \tau^{1 / 4}}-\frac{\cos \Psi_{0}}{R_{0}(\tau)}\right)-\left(4 \beta+\tau^{-1}\right) \frac{\Psi}{4}\right] \tau^{-3 / 4}
\end{aligned}
$$

For system (9) we can construct Lyapunov function [20]. Its construction is based on the asymptotics for Hamiltonian $H(R, \Psi, t)$ and additional term $G(R, \Psi, t)$ as $t \rightarrow \infty$ and in the vicinity of the equilibrium as $\rho=\sqrt{R^{2}+\Psi^{2}} \rightarrow 0$.

In the Hamiltonian we can select a positive definite quadratic form as the main term of the asymptotics:

$$
H(R, \Psi, t)=\frac{R^{2}}{2}+\frac{\Psi^{2}}{2}+\mathcal{O}\left(\rho^{3}\right)+\mathcal{O}\left(\rho^{2}\right) \mathcal{O}\left(t^{-1 / 7}\right), \quad \rho \rightarrow 0, \quad t \rightarrow \infty .
$$

We observe that hereinafter asymptotic estimates $\mathcal{O}\left(\rho^{n}\right)$ or $\mathcal{O}\left(t^{-m}\right)(n, m=$ const $>0)$ are uniform in $(R, \Psi, t)$ in some domain $\mathcal{D}\left(\rho_{0}, t_{0}\right)$ :

$$
\mathcal{D}\left(\rho_{0}, t_{0}\right)=\left\{(R, \Psi, t): \rho<\rho_{0}, t>t_{0}\right\}, \quad \rho_{0}, t_{0}=\text { const }>0 .
$$

\footnotetext{
${ }^{1}$ in the formulae we employ variable $\tau$, at that, $\tau=z t^{4 / 7}$
} 
The partial derivatives of Hamiltonian $H(R, \Psi, t)$ have the following asymptotics:

$\partial_{R} H=R+\frac{\nu R^{2}}{2 \sqrt{\lambda}} \tau^{-1 / 4}+\mathcal{O}(\rho) \mathcal{O}\left(\tau^{-1 / 2}\right), \quad \partial_{\Psi} H=\sin \Psi+\mathcal{O}(\rho) \mathcal{O}\left(\tau^{-1 / 2}\right), \quad \partial_{t} H=\mathcal{O}\left(\rho^{2}\right) \mathcal{O}\left(\tau^{-2}\right)$.

Function $G(R, \Psi, t)$ tends to zero as $t \rightarrow \infty$ :

$$
G(R, \Psi, t)=\frac{g_{1}(\cos \Psi-1)}{2 \nu \lambda} \tau^{-1 / 4}-\frac{g_{1} R \cos \Psi}{2 \lambda^{3 / 2}} \tau^{-1 / 2}-\frac{b \Psi}{2 \nu \lambda^{1 / 2}}[1+\mathcal{O}(\rho)] \tau^{-3 / 4}+\mathcal{O}\left(\tau^{-1}\right)
$$

and $b=\beta\left(1+g_{1} / f_{1}\right)>0$.

The base of constructed Lyapunov function is Hamiltonian which is perturbed by additional terms decaying with different rates as $t \rightarrow \infty$

$$
\begin{aligned}
& V(R, \Psi, t)=H(R, \Psi, t)+V_{1}(R, \Psi, t)+V_{2}(R, \Psi, t)+V_{3}(R, \Psi, t) \\
& V_{1}(R, \Psi, t)=\frac{g_{1} R}{2 \nu \lambda}\left(\cos \Psi-1-\frac{R^{2}}{3}\right) \tau^{-1 / 4} \\
& V_{2}(R, \Psi, t)=\left[\frac{g_{1}^{2}}{4 \nu^{2} \lambda^{2}}\left(\frac{\sin ^{2} \Psi}{2}+\cos \Psi-1\right)+\frac{g_{1}}{4 \lambda^{3 / 2}}\left(\sin ^{2} \Psi-\frac{R^{4}}{4}\right)\right] \tau^{-1 / 2}, \\
& V_{3}(R, \Psi, t)=-\frac{b R \Psi}{4 \nu \lambda^{1 / 2}} \tau^{-3 / 4}
\end{aligned}
$$

The derivative of these functions calculated along system (9) reads as

$$
\begin{aligned}
\left.\frac{d H}{d t}\right|_{\text {(9) }}= & \frac{\partial H}{\partial t}+\frac{\partial H}{\partial \Psi} G=\frac{g_{1}}{2 \nu \lambda} \sin \Psi(\cos \Psi-1) \tau^{-1 / 4}-\frac{g_{1}}{2 \lambda^{3 / 2}} R \sin \Psi \cos \Psi \tau^{-1 / 2} \\
& \quad-\frac{b}{2 \nu \lambda^{1 / 2}} \Psi^{2} \tau^{-3 / 4}+\mathcal{O}\left(\rho^{3}\right) \mathcal{O}\left(\tau^{-3 / 4}\right)+\mathcal{O}\left(\rho^{2}\right) \mathcal{O}\left(\tau^{-1}\right), \\
\left.\frac{d V_{1}}{d t}\right|_{\text {(9) }}= & -\frac{g_{1}}{2 \nu \lambda} \sin \Psi(\cos \Psi-1) \tau^{-1 / 4}-\frac{g_{1}}{4 \lambda^{3 / 2}} R \sin \Psi\left[R^{2}+\frac{g_{1}}{\nu^{2} \lambda^{1 / 2}}(\cos \Psi-1)\right] \tau^{-1 / 2} \\
& +\mathcal{O}\left(\rho^{3}\right) \mathcal{O}\left(\tau^{-3 / 4}\right)+\mathcal{O}\left(\rho^{2}\right) \mathcal{O}\left(\tau^{-1}\right), \\
\left.\frac{d V_{2}}{d t}\right|_{\text {(9) }}= & \frac{g_{1}}{2 \lambda^{3 / 2}} R \sin \Psi \cos \Psi \tau^{-1 / 2}+\frac{g_{1}}{4 \lambda^{3 / 2}} R \sin \Psi\left[R^{2}+\frac{g_{1}}{\nu^{2} \lambda^{1 / 2}}(\cos \Psi-1)\right] \tau^{-1 / 2} \\
& +\mathcal{O}\left(\rho^{3}\right) \mathcal{O}\left(\tau^{-3 / 4}\right)+\mathcal{O}\left(\rho^{2}\right) \mathcal{O}\left(\tau^{-1}\right), \\
\left.\frac{d V_{3}}{d t}\right|_{\text {(9) }}= & -\frac{b}{4 \nu \lambda^{1 / 2}}\left(R^{2}-\Psi^{2}\right) \tau^{-3 / 4}+\mathcal{O}\left(\rho^{3}\right) \mathcal{O}\left(\tau^{-3 / 4}\right)+\mathcal{O}\left(\rho^{2}\right) \mathcal{O}\left(\tau^{-1}\right) .
\end{aligned}
$$

It implies the expression for the derivative of function $V(R, \Psi, t)$, which happens to have a sign-definite leading term in the asymptotics:

$$
\left.\frac{d V}{d t}\right|_{\text {(9) }}=-\frac{\omega}{2} t^{-3 / 7}\left(R^{2}+\Psi^{2}\right)+\mathcal{O}\left(\rho^{3}\right) \mathcal{O}\left(t^{-3 / 7}\right)+\mathcal{O}\left(\rho^{2}\right) \mathcal{O}\left(t^{-4 / 7}\right),
$$

$\omega=b\left(2 \nu \lambda^{1 / 2} z^{3 / 4}\right)^{-1}>0$. Since the estimates in the last expression can be arbitrarily small, for each $\sigma>0$ there exist constants $\rho_{1}>0, t_{1}>0$ such that in neighborhood $\mathcal{D}\left(\rho_{1}, t_{1}\right)$ the upper estimate

$$
\left.\frac{d V}{d t}\right|_{(9)} \leqslant-\frac{(\omega-\sigma)}{2} t^{-3 / 7}\left(R^{2}+\Psi^{2}\right), \quad \omega-\sigma>0,
$$

holds true. In the same way we obtain the estimates for function $V(R, \Psi, t): \forall \sigma>0$ there exist $\rho_{2}>0, t_{2}>0$ :

$$
\frac{(1-\sigma)}{2}\left(R^{2}+\Psi^{2}\right) \leqslant V(R, \Psi, t) \leqslant \frac{(1+\sigma)}{2}\left(R^{2}+\Psi^{2}\right)
$$


as $(R, \Psi, t) \in \mathcal{D}\left(\rho_{2}, t_{2}\right)$. We choose $\sigma \in(0, \min \{1, \omega\})$, the in the domain $\mathcal{D}\left(\rho_{0}, t_{0}\right), \rho_{0}=$ $\min \left(\rho_{1}, \rho_{2}\right), t_{0}=\max \left(t_{1}, t_{2}\right)$, we have the estimate

$$
\left.\frac{d V}{d t}\right|_{\text {(9) }} \leqslant-\omega_{0} t^{-3 / 7} V \leqslant 0, \quad \omega_{0}=\frac{\omega-\sigma}{1+\sigma}>0 .
$$

We fix a small $\epsilon>0$ and we define $\delta_{\epsilon}=\epsilon \sqrt{1-\sigma} /(2 \sqrt{1+\sigma})$. Then Lyapunov function satisfies the inequalities:

$$
\sup _{\rho \leqslant \delta_{\epsilon}, t>t_{0}} V(R, \Psi, t) \leqslant(1+\sigma) \frac{\delta_{\epsilon}^{2}}{2} \leqslant(1-\sigma) \frac{\epsilon^{2}}{2} \leqslant \inf _{\rho=\epsilon, t>t_{0}} V(R, \Psi, t) .
$$

Since the derivative of Lyapunov function is negative, it follows that each trajectory $R(t), \Psi(t)$ with initial data $R^{2}\left(t_{0}\right)+\Psi^{2}\left(t_{0}\right) \leqslant \delta_{\epsilon}^{2}$ does not leave $\epsilon$-neighborhood of the equilibrium $R=0$, $\Psi=0$ as $t>t_{0}$. Moreover, it also follows from (14) that Lyapunov function decays on the trajectories of system (91):

$$
0 \leqslant V(R(t), \Psi(t), t) \leqslant C \exp \left(-\frac{7 \omega_{0}}{4} t^{4 / 7}\right), \quad t>t_{0} .
$$

Constant $C>0$ depends on the trajectory. By means of change of variables (8) we obtain estimates for the solutions to system (10) as $\tau>\tau_{0}, \tau_{0}=z t_{0}^{4 / 7}$ :

$$
\left|r(\tau)-R_{0}(\tau)\right| \leqslant \nu \tau^{1 / 4} \sqrt{\frac{2 C}{1-\sigma}} \exp \left(-\frac{7 \omega_{0}}{8 z} \tau\right), \quad\left|\psi(\tau)-\Psi_{0}(\tau)\right| \leqslant \sqrt{\frac{2 C}{1-\sigma}} \exp \left(-\frac{7 \omega_{0}}{8 z} \tau\right) .
$$

These inequalities imply the asymptotic stability of solution $R_{0}(\tau), \Psi_{0}(\tau)$.

Remark 1. In applications functions $f(\tau)$ and $g(\tau)$ are usually equal. In this case the statement of theorem becomes unconditional since $1+g_{1} / f_{1} \equiv 2>0$.

\section{StABILITY UNDER PERSISTENT PERTURBATIONS}

In this section we study the stability of solution $R_{0}(\tau), \Psi_{0}(\tau)$ w.r.t. persistent perturbations. Together with equations (11) we consider the perturbed system:

$$
\frac{d r}{d \tau}=(f(\tau)+\mu \xi) \sin \psi-\beta r, \quad r\left[\frac{d \psi}{d \tau}-r^{2}+\lambda \tau+\mu \varphi\right]=(g(\tau)+\mu \eta) \cos \psi .
$$

Functions $\xi(r, \psi, \tau), \eta(r, \psi, \tau), \varphi(r, \psi, \tau)$ correspond to perturbations, $\mu$ is a small parameter. Let us give the definition of stability we shall use in this section.

Definition 1. Solution $R_{0}(\tau), \Psi_{0}(\tau)$ of system (11) is stable w.r.t. persistent perturbations $\mathcal{B}$ if $\forall \epsilon>0 \exists \delta_{\epsilon}, \Delta_{\epsilon}>0$ :

$$
\forall \tau_{0}>0 \quad \forall \varrho_{0}, \phi_{0}: \quad\left|\varrho_{0}-R_{0}\left(\tau_{0}\right)\right| \leqslant \delta_{\epsilon}, \quad\left|\phi_{0}-\Psi_{0}\left(\tau_{0}\right)\right| \leqslant \delta_{\epsilon},
$$

$\forall|\mu|<\Delta_{\epsilon}, \forall(\xi, \eta, \varphi) \in \mathcal{B}$ solution $r_{\mu}(\tau), \psi_{\mu}(\tau)$ of perturbed equations (15) with initial data $\left.r_{\mu}\right|_{\tau=\tau_{0}}=\varrho_{0},\left.\psi_{\mu}\right|_{\tau=\tau_{0}}=\phi_{0}$ satisfy the inequalities

$$
\left|r_{\mu}(\tau)-R_{0}(\tau)\right| \tau^{-1 / 4}<\epsilon, \quad\left|\psi_{\mu}(\tau)-\Psi_{0}(\tau)\right|<\epsilon \quad \forall \tau>\tau_{0} .
$$

A class of perturbations $\mathcal{B}$, under which the autoresonance is stable, will be called admissible.

We observe that Definition 11weakens slightly a classical definition of stability [21, 22] because of the presence of the factor $\tau^{-1 / 4}$ in the inequality for the amplitude. This inequality can be regarded as the estimate for the norm in the space of continuous functions with the weight $\tau^{-1 / 4}$ :

$$
\|u(\tau)\|_{\mathcal{C}^{1 / 4}}=\sup _{\tau>\tau_{0}}|u(\tau)| \tau^{-1 / 4}
$$


On the other hand, the stability by Definition 1 is in some sense stronger than the stability w.r.t. part of variables [23], for which one needs the smallness of deviations only for some of the components of solution. The considered case corresponds to $\psi$-stability. Thus, for the stability we need to keep the rate of growth for the leading term of the asymptotics for the amplitude of perturbed solutions.

Remark 2. It follows from [3] that if $1+f_{1} / g_{1}>0$, then there exists a two-parametric family of solutions to equations (1) with the asymptotics $r(\tau)=\sqrt{\lambda \tau}+\mathcal{O}\left(\tau^{1 / 4}\right), \psi(\tau)=o(1)$ as $\tau \rightarrow \infty$. Perturbed system (15) can also have autoresonance solutions with such amplitude. This is why the presence of the factor $\tau^{-1 / 4}$ in the estimate for the amplitude in the definition of stability seems to be quite reasonable.

From the practical point of view, the above definition allows us to describe a wider class of perturbations under which the capture into autoresonance occurs.

Let $a, b, c=$ const $\geqslant 0$. We define the class of perturbations $\mathcal{P}_{a, b, c}$ as a set of functions $(\xi, \eta, \varphi)$ with a finite quantity

$$
\sup _{(r, \psi) \in \mathbb{R}^{2}, \tau>0}|\xi(r, \psi, \tau)| \tau^{-a}+|\eta(r, \psi, \tau)| \tau^{-b}+|\varphi(r, \psi, \tau)| \tau^{-c}<\infty .
$$

For each $m>0$ we define also $\mathcal{P}_{a, b, c}^{m}$ as the subset of functions $(\xi, \eta, \varphi) \in \mathcal{P}_{a, b, c}$ satisfying the inequality

$$
|\xi(r, \psi, \tau)| \tau^{-a}+|\eta(r, \psi, \tau)| \tau^{-b}+|\varphi(r, \psi, \tau)| \tau^{-c} \leqslant m, \quad \forall(r, \psi) \in \mathbb{R}^{2}, \tau>0 .
$$

The study of stability for solution $R_{0}(\tau), \Psi_{0}(\tau)$ is reduced to the stability of the equilibrium $(0 ; 0)$ of system (9) under persistent perturbations. In order to do it, in perturbed equations (15) we make the change of variables (8) and the system becomes

$$
\frac{d R}{d t}=-\partial_{\Psi} H(R, \Psi, t)+\mu p(R, \Psi, t), \quad \frac{d \Psi}{d t}=\partial_{R} H(R, \Psi, t)+G(R, \Psi, t)+\mu q(R, \Psi, t) .
$$

Hamiltonian $H(R, \Psi, t)$ and function $G(R, \Psi, t)$ are determined by formulae (10) and (11). Persistent perturbations of system (9) are described by functions $p(R, \Psi, t)$ and $q(R, \Psi, t)$ :

$$
p(R, \Psi, t)=-\frac{\hat{\xi}}{z f_{1}} t^{-4 / 7}, \quad q(R, \Psi, t)=-\frac{\nu}{z^{3 / 4} f_{1}}\left(\frac{\hat{\eta} \cos \left(\Psi+\Psi_{0}\right)}{R_{0}+\nu R z^{1 / 4} t^{1 / 7}}-\hat{\varphi}\right) t^{-3 / 7}
$$

Functions $\hat{\xi}(R, \Psi, t), \hat{\eta}(R, \Psi, t), \hat{\varphi}(R, \Psi, t)$ appears as a result of substituting (8) into $\xi(r, \psi, \tau)$, $\eta(r, \psi, \tau), \varphi(r, \psi, \tau)$, respectively, for instance,

$$
\hat{\xi}(R, \Psi, t)=\xi\left(R_{0}(\tau)+\nu R \tau^{1 / 4}, \Psi_{0}(\tau)+\Psi, \tau\right), \quad \tau=z t^{4 / 7} .
$$

We consider the auxiliary problem on stability of the trivial solution to system (9) under persistent perturbations $(p, q)$. We introduce a class $\mathcal{K}$ of perturbations of system (9) as the set of functions $(p, q)$ satisfying

$$
\sup _{(R, \Psi) \in \mathbb{R}^{2}, t>0}|p(R, \Psi, t)| t^{3 / 7}+|q(R, \Psi, t)| t^{3 / 7}<\infty .
$$

For each fixed $m>0$, the set of functions $(p, q) \in \mathcal{K}$ satisfying the estimate

$$
|p(R, \Psi, t)| t^{3 / 7} \leqslant m, \quad|q(R, \Psi, t)| t^{3 / 7} \leqslant m \quad \forall(R, \Psi) \in \mathbb{R}^{2}, t>0,
$$

is denoted by $\mathcal{K}^{m}$.

In studying systems (9) and (16) we employ the classical definition of stability [21]. 
Definition 2. Solution $R(t) \equiv 0, \Psi(t) \equiv 0$ of system (9) is stable w.r.t. persistent perturbations $\mathcal{K}$ if $\forall \epsilon>0 \exists \delta_{\epsilon}, \Delta_{\epsilon}>0$ :

$$
\forall t_{0}>0 \quad \forall \varrho_{0}, \phi_{0}: \varrho_{0}^{2}+\phi_{0}^{2} \leqslant \delta_{\epsilon}^{2}, \quad \forall|\mu|<\Delta_{\epsilon}, \quad \forall(p, q) \in \mathcal{K},
$$

solution $R_{\mu}(t), \Psi_{\mu}(t)$ to perturbed equations (16) with initial data $\left.R_{\mu}\right|_{t=t_{0}}=\varrho_{0},\left.\Psi_{\mu}\right|_{t=t_{0}}=\phi_{0}$ satisfies the inequality:

$$
R_{\mu}^{2}(t)+\Psi_{\mu}^{2}(t)<\epsilon^{2} \quad \forall t>t_{0}
$$

We have

Lemma 1. If the coefficients of system (9) satisfy inequality $1+g_{1} / f_{1}>0$, then $\forall m>0$ the trivial solution $R(t) \equiv 0, \Psi(t) \equiv 0$ is stable under persistent perturbations $(p, q) \in \mathcal{K}^{m}$.

Proof. The proof is based on Lyapunov function (12) constructed in Theorem 1 for unperturbed system (9). We calculate the total derivative of function $V(R, \Psi, t)$ along the perturbed system (16):

$$
\left.\frac{d V}{d t}\right|_{(16)}=\left.\frac{d V}{d t}\right|_{\text {(9) }}+\mu\left(p \partial_{R} V+q \partial_{\Psi} V\right) .
$$

The first term in the right hand side of this identity satisfies estimate (14) in domain $\mathcal{D}\left(\rho_{0}, t_{0}\right)$ with constants $\sigma, \omega, \omega_{0}>0$. Partial derivatives of function $V$ are bounded in the vicinity of zero $R=0, \Psi=0$ as $t>t_{0}:\left|\partial_{R} V\right| \leqslant \ell,\left|\partial_{\Psi} V\right| \leqslant \ell$. We fix parameters $\epsilon>0$ and $m>0$ and let $\epsilon$ be sufficiently small. We define $\Delta_{\epsilon}=\epsilon^{2} \omega_{0}(1-\sigma)^{2} /(32 m \ell(1+\sigma))$. Then as $|\mu|<\Delta_{\epsilon}$ for an appropriate choice of $\delta_{\epsilon} \in\left(0, \rho_{0}\right)$ the inequalities

$$
\left.\frac{d V}{d t}\right|_{(16)} \leqslant-\omega_{0} t^{-3 / 7}\left[V-\frac{\epsilon^{2}(1-\sigma)^{2}}{16(1+\sigma)}\right]<0, \quad \delta_{\epsilon}=\frac{\epsilon}{2} \sqrt{\frac{1-\sigma}{1+\sigma}}<\epsilon
$$

hold true in domain $\mathcal{D}\left(\rho_{0}, t_{0}\right) \backslash \overline{\mathcal{D}\left(\delta_{\epsilon}, t_{0}\right)}$. Estimates (13) for Lyapunov function imply the inequalities

$$
\sup _{\rho \leqslant \delta_{\epsilon}, t>t_{0}} V(R, \Psi, t) \leqslant(1+\sigma) \frac{\delta_{\epsilon}^{2}}{2}<(1-\sigma) \frac{\epsilon^{2}}{2} \leqslant \inf _{\rho=\epsilon, t>t_{0}} V(R, \Psi, t)
$$

Together with the negativeness of the total derivative of function $V(R, \Psi, t)$ it yields that for each $T_{0}>t_{0}$, solution to system (16) with initial conditions $R_{\mu}^{2}\left(T_{0}\right)+\Psi_{\mu}^{2}\left(T_{0}\right)=\delta_{\epsilon}^{2}$ stays in the $\epsilon$-neighborhood of zero, i.e., $R_{\mu}^{2}(t)+\Psi_{\mu}^{2}(t)<\epsilon^{2}$ as $t>T_{0}$.

For the trajectories of perturbed system (16) with initial conditions $R_{\mu}\left(T_{0}\right), \Psi_{\mu}\left(T_{0}\right)$ in the circle $\rho<\delta_{\epsilon}$, where the negativeness of the derivative of Lyapunov function is not ensured, there are two possibilities: either $R_{\mu}^{2}(t)+\Psi_{\mu}^{2}(t)<\delta_{\epsilon}^{2}$ for each $t>T_{0}$ or there exists $t_{\epsilon}>T_{0}$ such that $R_{\mu}^{2}\left(t_{\epsilon}\right)+\Psi_{\mu}^{2}\left(t_{\epsilon}\right)=\delta_{\epsilon}^{2}$. In the latter case estimates (18) and (19) imply that $R_{\mu}^{2}(t)+\Psi_{\mu}^{2}(t)<\epsilon^{2}$ as $t>t_{\epsilon}$.

Thus, we have proven the stability of the trivial solution in the neighborhood of the infinity $t>t_{0}$. The stability on a finite segment $0 \leqslant t \leqslant t_{0}$ follows from the theorem on continuity of solution to Cauchy problem w.r.t. the parameters of equations (cf. [14]).

Let us specify class of perturbations $\mathcal{P}$ of system (1). We choose parameters $a=1 / 4, b=1 / 2$, $c=0$, then functions $p$ and $q$ determined by formulae (17) belong to class $\mathcal{K}$.

Theorem 2. Suppose that the coefficients of system (1) satisfy inequality $1+g_{1} / f_{1}>0$. Then $\forall m>0$ solution $R_{0}(\tau), \Psi_{0}(\tau)$ with asymptotics (7) is stable w.r.t. persistent perturbations $(\xi, \eta, \varphi) \in \mathcal{P}_{1 / 4,1 / 2,0}^{m}$ :

$$
|\xi(r, \psi, \tau)| \leqslant m \tau^{1 / 4}, \quad|\eta(r, \psi, \tau)| \leqslant m \tau^{1 / 2}, \quad|\varphi(r, \psi, \tau)| \leqslant m, \quad \tau>0 .
$$


Proof. We observe that functions $\hat{\xi}(R, \Psi, t), \hat{\eta}(R, \Psi, t), \hat{\varphi}(R, \Psi, t)$ satisfy the estimates:

$$
|\hat{\xi}(R, \Psi, t)| \leqslant \hat{m} t^{1 / 7}, \quad|\hat{\eta}(R, \Psi, t)| \leqslant \hat{m} t^{2 / 7}, \quad|\hat{\varphi}(R, \Psi, t)| \leqslant \hat{m} \quad \forall(R, \Psi), t>t_{0},
$$

$\hat{m}=m \cdot \max \left\{1, z^{1 / 4}, z^{1 / 2}\right\}$. In view of the structure of functions $p(R, \Psi, t)$ and $q(R, \Psi, t)$ it implies the inequalities $|p(R, \Psi, t)| \leqslant M t^{-3 / 7},|q(R, \Psi, t)| \leqslant M t^{-3 / 7}$ with a positive constant $M=m z^{-3 / 4} \max \left\{1,|\nu|\left(1+\lambda^{-1 / 2}\right)\right\} /\left|f_{1}\right|$, i.e., $(p, q) \in \mathcal{K}$. Then in accordance with Lemma 1 the trivial solution to system (9) is stable w.r.t. persistent perturbations (in the sense of Definition 21) and for the trajectories of system (16) with initial conditions in the vicinity of the infinity the estimate

$$
R_{\mu}^{2}(t)+\Psi_{\mu}^{2}(t)<\epsilon^{2}
$$

holds true as $t>0$. Therefore, $\forall \tau_{0}>0$ in view of change (8) the solutions to system (15) with initial conditions close to $R_{0}\left(\tau_{0}\right), \Psi_{0}\left(\tau_{0}\right)$ the inequalities

$$
\left|r_{\mu}(\tau)-R_{0}(\tau)\right| \tau^{-1 / 4}<\nu \epsilon, \quad\left|\psi_{\mu}(\tau)-\Psi_{0}(\tau)\right|<\epsilon, \quad \tau>\tau_{0},
$$

hold true. It implies the stability of solution $R_{0}(\tau), \Psi_{0}(\tau)$ in the sense of Definition 1 .

\section{StABility ON ASYMPtoticAlLy LONG TIME INTERVAL}

In the previous section we have described the class of perturbations under which the solution to system (11) with asymptotics (7) is stable for each $\tau>0$. However, to study the capture into autoresonance by the averaged equations it is sufficient to restrict ourselves by a finite time interval on which the mathematical model is still appropriate [1]: $0<\tau \leqslant \mathcal{O}\left(\varepsilon^{-1 / 3}\right)$, where $0<\varepsilon \ll 1$ is a small parameter of pumping in the original equation, see, for instance, (2). In the subsequent time the capture into autoresonance is described by other equations [24]. Hence, in the considered case, a more appropriate problem is on the stability under persistent perturbation on a finite but an asymptotically long time interval $0<\tau \leqslant \mathcal{O}\left(|\mu|^{-\varkappa}\right)$, where $|\mu| \ll 1$ is the perturbation parameter. A similar formulation was considered, for instance, in [13]. It is obvious that the stability of solution on the half-line under the perturbations in class $\mathcal{P}$ implies the stability on each finite interval. The attempt to restrict the considered time interval is motivated by the hope to find weaker conditions (in comparison with (201) for admissible classes of perturbations. A similar approach happened to be quite successful in analyzing stability for dynamical systems perturbed by "white noise" [25].

Let us formulate the definition of stability.

Definition 3. Solution $R_{0}(\tau), \Psi_{0}(\tau)$ to system (1) is stable w.r.t. persistent perturbations $\mathcal{B}$ on an asymptotically long time interval if there exists $\varkappa>0$ such that $\forall \epsilon>0 \exists \delta_{\epsilon}, \Delta_{\epsilon}>0$ :

$$
\forall \varrho_{0}, \phi_{0}: \quad\left|\varrho_{0}-R_{0}(0)\right| \leqslant \delta_{\epsilon}, \quad\left|\phi_{0}-\Psi_{0}(0)\right| \leqslant \delta_{\epsilon}, \quad \forall|\mu|<\Delta_{\epsilon}, \quad \forall(\xi, \eta, \varphi) \in \mathcal{B}
$$

solution $r_{\mu}(\tau), \psi_{\mu}(\tau)$ to perturbed equations (15) with initial data $\left.r_{\mu}\right|_{\tau=0}=\varrho_{0},\left.\quad \psi_{\mu}\right|_{\tau=0}=\phi_{0}$ satisfy the inequalities

$$
\left|r_{\mu}(\tau)-R_{0}(\tau)\right| \tau^{-1 / 4}<\epsilon, \quad\left|\psi_{\mu}(\tau)-\Psi_{0}(\tau)\right|<\epsilon
$$

as $0<\tau \leqslant \mathcal{O}\left(|\mu|^{-\varkappa}\right)$.

We shall consider class of perturbations $\mathcal{P}_{a, b, c}$ introduced in the previous section but with other parameters: $a=1+\vartheta, b=5 / 4+\vartheta, c=3 / 4+\vartheta, \vartheta \geqslant 0$.

Theorem 3. Suppose that the coefficients in system (11) satisfy the inequality $1+g_{1} / f_{1}>0$. Then $\forall m>0, \vartheta \geqslant 0$ solution $R_{0}(\tau), \Psi_{0}(\tau)$ with asymptotics (7) is stable w.r.t. persistent perturbations $(\xi, \eta, \varphi) \in \mathcal{P}_{1+\vartheta, 5 / 4+\vartheta, 3 / 4+\vartheta}^{m}$ :

$$
|\xi(r, \psi, \tau)| \leqslant m \tau^{1+\vartheta}, \quad|\eta(r, \psi, \tau)| \leqslant m \tau^{5 / 4+\vartheta}, \quad|\varphi(r, \psi, \tau)| \leqslant m \tau^{3 / 4+\vartheta}
$$


on an asymptotically long time interval $0<\tau \leqslant \mathcal{O}\left(|\mu|^{-\varkappa}\right), 0<\varkappa<\varkappa_{0}, \varkappa_{0}=4 /(4 \vartheta+3)$.

Proof. As in the proof of Theorem 2, we first study perturbed system (16) and then we conclude on the stability of solutions to original equations (1).

Under above restrictions and in view of (17) functions $p(R, \Psi, t)$ and $q(R, \Psi, t)$ satisfy the estimates $|p(R, \Psi, t)| \leqslant M t^{4 \vartheta / 7},|q(R, \Psi, t)| \leqslant M t^{4 \vartheta / 7}$ with a positive constant $M=$ $m z^{\vartheta} \max \left\{1,|\nu|\left(1+\lambda^{-1 / 2}\right)\right\} /\left|f_{1}\right|$. Let us calculate the derivative of Lyapunov function (12) along system (16):

$$
\left.\frac{d V}{d t}\right|_{(16)}=\left.\frac{d V}{d t}\right|_{(9)}+\mu\left(p \partial_{R} V+q \partial_{\Psi} V\right) .
$$

In domain $\mathcal{D}\left(\rho_{0}, t_{0}\right)$ the derivative satisfies the estimate

$$
\left.\frac{d V}{d t}\right|_{[16)} \leqslant-\omega_{0} t^{-3 / 7} V+2|\mu| \ell M t^{4 \vartheta / 7} .
$$

The first term in the right hand side emerges because of estimate (14) for the total derivative of function $V(R, \Psi, t)$. The boundedness of perturbations $p(R, \Psi, t), q(R, \Psi, t)$ and partial derivatives of function $V(R, \Psi, t)$ in domain $\mathcal{D}\left(\rho_{0}, t_{0}\right):\left|\partial_{R} V\right| \leqslant \ell,\left|\partial_{\Psi} V\right| \leqslant \ell$ lead us to the other term in the estimate. We fix $\epsilon>0, m>0,0<\kappa<7 /(4 \vartheta+3)$ and choose

$$
\Delta_{\epsilon}=\left(2 t_{0}\right)^{-(4 \vartheta+3) / \kappa_{0}}\left[\frac{\epsilon^{2} \omega_{0}(1-\sigma)^{2}}{32 M \ell(1+\sigma)}\right]^{7 / \kappa_{0}}, \quad \kappa_{0}=7-(4 \vartheta+3) \kappa>0 .
$$

Then as $|\mu|<\Delta_{\epsilon}$ and for an appropriate choice of $\delta_{\epsilon} \in\left(0, \rho_{0}\right)$ the estimates

$$
\left.\frac{d V}{d t}\right|_{(16)} \leqslant-\omega_{0} t^{-3 / 7}\left[V-(1-\sigma) \frac{\delta_{\epsilon}^{2}}{4}\right]<0, \quad \delta_{\epsilon}=\frac{\epsilon}{2} \sqrt{\frac{1-\sigma}{1+\sigma}}<\epsilon
$$

hold true in the bounded domain $\delta_{\epsilon}<\rho<\rho_{0}, 0<t-t_{0} \leqslant t_{0}|\mu|^{-\kappa}$. We note that for chosen $\delta_{\epsilon}$ Lyapunov function satisfies inequalities (19). Together with the negativeness of total derivative of function $V(R, \Psi, t)$ it follows that each solution to system (16) with initial data $R_{\mu}^{2}\left(t_{0}\right)+\Psi_{\mu}^{2}\left(t_{0}\right)=\delta_{\epsilon}^{2}$ stays in $\epsilon$-neighborhood of zero, i.e., $R_{\mu}^{2}(t)+\Psi_{\mu}^{2}(t)<\epsilon^{2}$ as $0 \leqslant t-t_{0} \leqslant$ $t_{0}|\mu|^{-\kappa}$.

In circle $\rho<\delta_{\epsilon}$ the negativeness of the total derivative of function $V(R, \Psi, t)$ is not ensured. This is why the trajectories of system (16) with initial data $R_{\mu}^{2}\left(t_{0}\right)+\Psi_{\mu}^{2}\left(t_{0}\right)<\delta_{\epsilon}^{2}$ either stay bounded $R_{\mu}^{2}(t)+\Psi_{\mu}^{2}(t)<\delta_{\epsilon}^{2}$ on the interval $0 \leqslant t-t_{0} \leqslant t_{0}|\mu|^{-\kappa}$ or there exists $t_{\epsilon}: 0<t_{\epsilon}-t_{0}<$ $t_{0}|\mu|^{-\kappa}$ such that $R_{\mu}^{2}\left(t_{\epsilon}\right)+\Psi_{\mu}^{2}\left(t_{\epsilon}\right)=\delta_{\epsilon}^{2}$. In the latter case the estimates (18) and (19) imply that $R_{\mu}^{2}(t)+\Psi_{\mu}^{2}(t)<\epsilon^{2}$ as $t \in\left[t_{\epsilon}, t_{0}+t_{0}|\mu|^{-\kappa}\right]$. Thus, $R_{\mu}^{2}(t)+\Psi_{\mu}^{2}(t)<\epsilon^{2}$ as $0 \leqslant t-t_{0} \leqslant t_{0}|\mu|^{-\kappa}$.

By means of change of variables (8) we obtain the estimates for the solutions to original system (15):

$$
\left|r_{\mu}(\tau)-R_{0}(\tau)\right| \tau^{-1 / 4}<\nu \epsilon, \quad\left|\psi_{\mu}(\tau)-\Psi_{0}(\tau)\right|<\epsilon
$$

as $0 \leqslant \tau-\tau_{0} \leqslant \mathcal{O}\left(|\mu|^{-4 \kappa / 7}\right)$. Theorem on continuity of solution to Cauchy problem w.r.t. the parameters of equations implies the stability of solution to equation for $\tau \in\left(0 ; \tau_{0}\right)$. Therefore, $\forall m>0$ and $\vartheta \geqslant 0$ solution $R_{0}(\tau), \Psi_{0}(\tau)$ to system (11) is stable under persistent perturbations on an asymptotically long time interval $0<\tau \leqslant \mathcal{O}\left(|\mu|^{-\varkappa}\right), \forall \varkappa \in\left(0 ; \varkappa_{0}\right)$ uniformly in $(\xi, \eta, \varphi) \in$ $\mathcal{P}_{m}$.

Remark 3. Theorem [3] and formulae (6) imply the stability of autoresonance solutions to Duffing equations with external pumping (2). If $\mu=\varepsilon^{1 / 3}, h(s ; \varepsilon)=\varepsilon^{5 / 6} s^{5 / 4}, \theta(s ; \varepsilon)=\varepsilon^{4 / 3} s^{2}$ (that corresponds to $\vartheta=1 / 4$ ), perturbed equation (5) have trajectories captured into autoresonance as $s \leqslant \mathcal{O}\left(\varepsilon^{-5 / 6}\right)$. 


\section{Conclusion}

We study a system of model equations describing the initial stage of the capture into autoresonance in nonlinear oscillating systems with dissipation. We prove the Lyapunov stability of solutions with growing amplitude $r(\tau) \approx \sqrt{\lambda \tau}$ and under persistent perturbations. We describe admissible classes of perturbations. The obtained results give a hope for the existence of rather long autoresonance regimes in real physical processes. The question on influence of random perturbations on the capture into autoresonance in systems with dissipation remains open. This subject requires a special attention and will be discussed in a separate work.

The author thanks L.A. Kalyakin for useful discussion and valuable comments.

\section{BIBLIOGRAPHY}

1. L.A. Kalyakin. Asymptotic analysis of autoresonance models // Uspekhi Matem. Nauk. 63:5(383), 3-72 (2008). [Russ. Math. Surv. 63:5, 791-857 (2008).]

2. O.M. Kiselev, S.G. Glebov. The capture into parametric autoresonance // Nonl. Dynam. 48:1-2, 217-230 (2007).

3. L.A. Kalyakin, M.A. Shamsutdinov. Autoresonant asymptotics in an oscillating system with weak dissipation // Theor. Matem. Fiz. 160:1, 102-111 (2009). [Theor. Math. Phys. 160:1, 960-967 (2009).]

4. N.N. Bogolyubov and Yu.A.Mitropol'skǐ. Asymptotics methods in theory of nonlinear oscillations. Nauka, Moscow (1974). [Gordon and Breach, New York (1962).]

5. G.M. Zaslavskii, R.Z. Sagdeev. Introduction to nonlinear physics. From the pendulum to turbulence and chaos. Nauka, Moscow (1988). (in Russian).

6. V.I. Veksler. A new method of accelerating relativistic particles // Dokl. AN SSSR. 43:8, 346-248 (1944). (in Russian).

7. A.T. Sinclair. On the origin of the commensurabilities amongst the satellites of Saturn // Mon. Not. Royal Astron. Soc. 160:2, 169-187 (1972).

8. K.S. Golovanivsky. Hydromagnetic autoresonance with a variable frequency // Fizika Plasmy. 11:3, 295-299 (1985). (in Russian).

9. J. Fajans, L. Friedland. Autoresonant (nonstationary) excitation of pendulums. Plutinos, plasmas, and other nonlinear oscillators // Am. J. Phys. 69:10, 1096-1102 (2001).

10. S. Glebov, O. Kiselev, N. Tarkhanov. Autoresonance in a dissipative sytem // J. Phys. A. 43:21, id 215203 (2010).

11. A.N. Kuznetsov. Existence of solutions entering at a singular point of an autonomous system having a formal solution // Funkts. Anal. Pril. 23:4, 63-74 (1989). [Funct. Anal. Appl. 23:4, 308-317 (1989).]

12. L.A. Kalyakin. Existence theorems and estimates of solutions for equations of principal resonance // Sovrem. Mat. Prilozh. 85:4, 73-83 (2012). [J. Math. Sci. 200:1, 82-95 (2014).]

13. M.M. Khapaev. Asymptotic methods and stability in theory of nonlinear oscillations. Vysshaya Shkola, Moscow (1988). (in Russian).

14. V.V. Nemystkii, V.V. Stepanov. Qualitative theory of differential equations. Editorial, Moscow (2004). (in Russian).

15. R.Z. Khas'minskil. Stability of systems of differential equations under random perturbations of their parameters. Nauka, Moscow (1969).

16. L.A Kalyakin, O.A. Sultanov. Stability of autoresonance models // Differ. Uravn. 49:3, 279-293 (2013). [Differ. Equat. 49:3, 267-281 (2013).]

17. O.A. Sultanov. Stability of autoresonance models under perturbation bounded in the mean // Trudy IMM UrO RAN. 19:3, 274-283 (2013). (in Russian).

18. O.A. Sultanov. Stability of autoresonance models subject to random perturbations for systems of nonlinear oscillation equations // Zh. Vychisl. Mat. Mat. Fiz. 54:11, 65-79 (2014). [Comp. Math. Math. Phys. 54:1, 59-73 (2014).] 
19. A.A. Kolomensky, A.N. Lebedev. Theory of cyclic accelerators. GIFML, Moscow (1962). [North Holland, Amsterdam (1969).]

20. O.A. Sultanov. Lyapunov functions for nonautonomous systems close to Hamiltonian // Ufimskij Matem. Zhurn. 2:4, 88-98 (2010). (in Russian).

21. I.G. Malkin. Theory of stability of motion. GITTL, Moscow-Leningrad (1952). [Theorie der Stabilität einer Bewegung. Verlag R. Oldenbourg, München (1959). (in German).]

22. N.N. Krasovsky. Some problems in theory of motion stability. Fizmatgiz, Moscow (1959). (in Russian).

23. V.V. Rumyantsev. A.S. Oziraner. Stability and stabilization of motion with respect to a part of the variables. Nauka, Moscow (1987).

24. R.N. Garifullin. Asymptotic solution to the problem of autoresonance: Outer expansion // Zhurn. Vychisl. Matem. Matem. Fiz. 46:9, 1605-1616 (2006). [Comp. Math. Math. Phys. 46:9, 1526-1538 (2009).]

25. L. Kalyakin. Stability under persistent perturbation by white noise // J. Phys. Conf. Ser. 482:1, id 012019 (2014).

Oskar Anvarovich Sultanov,

Institute of Mathematics CC USC RAS,

Chernyshevskii str., 112,

450008, Ufa, Russia

E-mail: oasultanov@gmail.com 